\title{
Left internal mammary graft stenosis and restenosis following angioplasty and stenting
}

\author{
C G C Spencer, G Y H Lip
}

\begin{abstract}
Coronary bypass grafts using the internal mammary artery usually have an excellent record of success and long term patency. We report a 42 year old man who initially presented with a history of atypical left sided chest pain, who had coronary artery bypass surgery for a severe stenosis in his proximal left anterior descending coronary artery (LAD) and moderate stenosis of his proximal circumflex artery, with his LIMA being grafted to his mid-LAD and a saphenous venous graft to the proximal LAD. He subsequently developed multiple stenoses in the LIMA graft which required coronary augioplasty and stenting, on more than one occasion, in view of very rapid restenosis within the LIMA graft.

(Heart 2001;86:e9)
\end{abstract}

Keywords: graft patency; left internal mammary artery grafts; restenosis; stenosis

A 42 year old white man had his first presentation with a history of atypical left sided chest pain in 1995 . He was a smoker and had a family history of ischaemic heart disease, with three brothers having suffered heart attacks before the age of 45 . His serum cholesterol was $4.6 \mathrm{mmol} / \mathrm{l}$ and total cholesterol to high density lipoprotein cholesterol ratio was 4.9. His ECG showed new lateral $\mathrm{T}$ wave inversion, although there was no significant rise in cardiac enzymes. Three days later he underwent coronary angiography, which showed a severe stenosis in his proximal left anterior descending coronary artery (LAD) and moderate stenosis of his proximal circumflex artery. Five days later he underwent coronary artery bypass surgery with his left internal mammary artery (LIMA) being grafted to his mid LAD and a saphenous venous graft grafted to the proximal LAD. One month after his operation, he was admitted to his local hospital with further left sided chest pain. Repeat coronary angiography showed a stenosis at the insertion of his LIMA graft into his LAD, which was felt to be the culprit lesion This was treated by percutaneous transluminal coronary angioplasty (PTCA) and the insertion of an intracoronary stent. $\mathrm{He}$ continued to have chest pain, and repeat angiography five days later showed a patent distal LIMA stent, but as there was a moderate stenosis in the proximal portion of his LIMA this was also treated by PTCA and stent deployment.

After two trouble free months he was admitted to our hospital with left sided chest pain radiating to his left arm. On this occasion, there were no significant ECG changes although his total serum creatine kinase was notably increased at $1147 \mathrm{iu} / \mathrm{dl}$ (normal < 150) with the creatine kinase $\mathrm{MB}$ (myocardial) fraction only $16 \mathrm{iU} / \mathrm{dl}(1.4 \%$, normal $<6 \%)$. However, because of continuing pain, he underwent coronary and graft angiography, which showed a patent saphenous vein graft grafted to the proximal LAD but a severe stenosis involving the insertion of the LIMA graft into the LAD and the LAD itself (fig 1). There was also a severe in-stent restenosis in the proximal portion of the LIMA graft, proximal to several intercostal branches.

PTCA to the proximal LIMA was attempted using a $3.0 \times 20 \mathrm{~mm}$ Worldpass balloon (Cordis, Miami, Florida, USA). Inflation of the balloon to 12 atm failed to dilate the LIMA graft stenosis but resulted in very severe, excruciating left sided chest pain, which was similar to that experienced by the patient at the time of his admission to hospital, with no concomitant ECG changes. The balloon was then

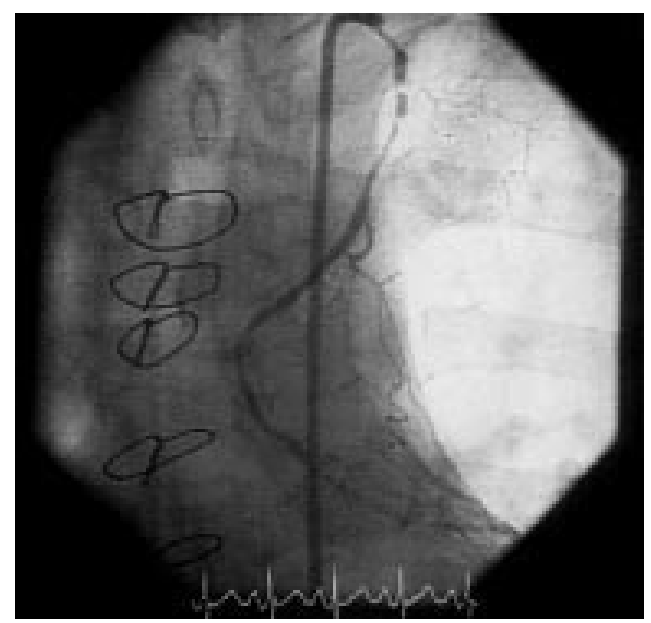

Figure 1 Severe stenosis involving the insertion of the left internal mammary artery (LIMA) graft into the left anterior descending coronary artery and the left anterior descending coronary artery itself. Note the severe in-stent restenosis in the proximal portion of the LIMA graft, proximal to several intercostal branches. 


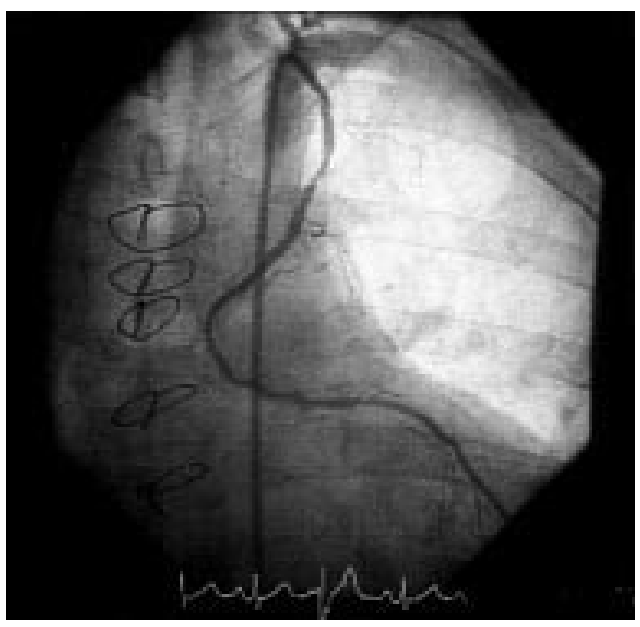

Figure 2 Post-PTCA, showing patent LIMA graft with good run off but mild residual proximal stenosis.

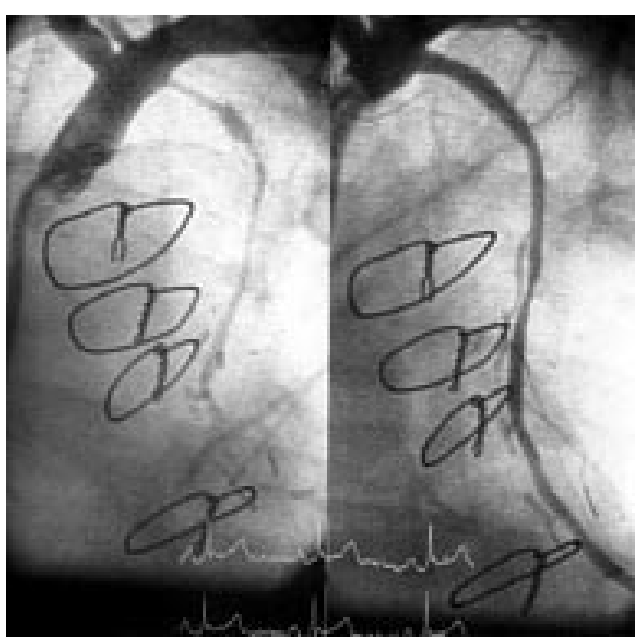

Figure 3 Restenosis in the proximal LIMA stent extending to the ostium of the LIMA, before (left) and after (right) PTCA.

advanced to the stenosis at the insertion of the LIMA into the LAD, which was successfully dilated, but inflation of the balloon in this position produced virtually no chest pain, in contrast to inflation of the balloon in the proximal LIMA. The proximal lesion was subsequently dilated to 18 atm using a $3.5 \times 10 \mathrm{~mm}$ Viva non-compliant balloon (Scimed, Boston Scientific, Galway, Ireland). The final result was a patent LIMA graft with good run off but mild residual proximal stenosis (fig 2).

The patient remained well and pain-free for two months but then started to get increasing chest pain, necessitating recurrent admissions to hospital. He was readmitted for repeat coronary angiography, which showed restenosis in the proximal LIMA stent extending to the ostium of the LIMA. PTCA to the ostium and proximal LIMA graft was successfully performed, again using a $3.0 \times 20 \mathrm{~mm}$ Worldpass balloon (fig 3).

\section{Discussion}

Although internal mammary artery grafts have an excellent record of success and long term patency, ${ }^{1}$, the course of our patient illustrates a number of the problems associated with these grafts.

The majority of patients requiring intervention for LIMA graft problems have stenosis at the site of insertion into the native coronary artery, which can be treated by angioplasty with a high degree of procedural success. ${ }^{2}$ Lesions in the proximal vessel appearing soon after surgery may be caused by kinking of the LIMA during surgical mobilisation ${ }^{3}$ or occasionally a surgical clip, ${ }^{4}$ both of which may be treated by angioplasty. Lastly, intercostal branches that have not been tied off may potentially cause problems. In the present case, much of the patient's chest pain may have originated from chest wall ischaemia, resulting in increased serum total creatine but not cardiac (MB) isoenzyme. The fact that the patient's pain could be reproduced by balloon inflation in the proximal LIMA but not in the distal vessel tends to confirm this hypothesis.

Finally, the patient re-presented with a long, stenotic segment at the ostium and proximal segment of his LIMA graft, which was successfully treated with PTCA. In view of the excellent long term patency of LIMA grafts, ${ }^{1}$ such cases are unusual, and we are unaware of previous reports of such a rapid stenosis (and restenosis following PTCA) of the LIMA graft.

1 Tector AJ, Schmahl TM, Janson B, et al. The internal mammary artery graft: its longevity after coronary bypass.

2 Dimas AP, Arora RR, Whitlow PL, et al. Percutaneous transluminal angioplasty involving internal mammary artery grafts. Am Heart f 1991;122:423-9.

3 Rerkpattanapipat P, Ghassemi R, Ledley GS, et al. Use of stents to treat kinks causing obstruction in a left internal mammary artery graft. Cathet Cardiovasc Intervent 1999;46: 223-6.

4 Klein AL, Marquis JF, Higginson LA. Percutaneous transluminal angioplasty of a surgically obstructed left internal mammary artery graft. Cathet Cardiovasc Diagn 1988;14:46-8 